\title{
Altered processing of corollary discharge in thalamic lesion patients
}

\author{
Christian Bellebaum, ${ }^{1,4}$ Klaus-Peter Hoffmann, ${ }^{2,4}$ Benno Koch, ${ }^{3}$ Michael Schwarz ${ }^{3}$ and Irene Daum ${ }^{1,4}$ \\ ${ }^{1}$ Institute of Cognitive Neuroscience, Department of Neuropsychology, Faculty of Psychology and \\ ${ }^{2}$ Department of General Zoology and Neurobiology, Ruhr-University of Bochum, D-44780 Bochum, Germany \\ ${ }^{3}$ Department of Neurology, Klinikum Dortmund, Dortmund, Germany \\ ${ }^{4}$ International Graduate School of Neuroscience, Bochum, Germany
}

Keywords: event-related potentials, posterior parietal cortex, saccades, updating

\begin{abstract}
Accumulating evidence suggests that thalamic nuclei relay corollary discharge information of saccadic eye movements, enabling the visual system to update the representation of visual space. The present study aimed to explore the effect of thalamic lesions in humans on updating-related cortical processing. Event-related potentials were recorded while four patients with impairments in using corollary discharge information and 12 healthy control subjects performed a saccadic double-step task. In the experimental condition, which required the use of corollary discharge information, control subjects showed a pronounced positivity over the parietal cortex starting about $150 \mathrm{~ms}$ after first saccade onset, reflecting the updating process. In the patients, parietal processing related to updating was altered. Three patients showed evidence of reduced updating event-related potential effects, consistent with a unilateral deficit in using corollary discharge information. In two patients, the event-related potential topography differed significantly from the topography pattern observed in controls. Thalamic damage affects updating-related processing, presumably due to insufficient transfer of saccade-related information to parietal areas. This study thus provides further evidence for thalamic involvement in relaying corollary discharge information related to saccadic eye movements. Our data suggest that integration of corollary discharge and motor information occurs directly before the second saccade in a double-step task.
\end{abstract}

\section{Introduction}

Whenever we perform a saccadic eye movement, the retinal positions of objects in our environment change. To achieve a stable percept of the world, visual space must be updated. Thus, the retinal consequences of saccades must be accounted for by extraretinal signals, i.e. most probably corollary discharge signals of the motor command to move the eyes (Sperry, 1950; von Holst \& Mittelstaedt, 1950).

In monkeys, the medio-dorsal nucleus of the thalamus (MD) relays information about contralaterally directed saccades from the superior colliculus to the frontal eye field (FEF) (Sommer \& Wurtz, 2004a,b). In humans, impairments in updating visual space have been found following lesions in the central thalamus (Gaymard et al., 1994), including the nuclei of the internal medullary lamina, and lesions in the centro-medial thalamus (Versino et al., 2000). In a more recent study, a large population of thalamic lesion patients differing in lesion locations was studied using a saccadic double-step task (Bellebaum et al., 2005a) to assess the ability to use corollary discharge information of saccadic eye movements (Hallett \& Lightstone, 1976). MD lesions as well as lateral thalamic lesions, involving the ventrolateral nucleus of the thalamus (VL), were associated with visual space-updating impairments, with deficits after lateral lesions resembling those found in monkeys with MD lesions (Sommer \& Wurtz, 2004b; Bellebaum et al., 2005a). In monkeys and humans, the

Correspondence: Dr Christian Bellebaum, ${ }^{1}$ Institute of Cognitive Neuroscience, as above. E-mail: christian.bellebaum@rub.de

Received 5 May 2006, revised 21 July 2006, accepted 14 August 2006 posterior parietal cortex (PPC) has been shown to play an important role in integrating visual and saccade-related information (Duhamel et al., 1992a; Haarmeier et al., 1997; Heide \& Kompf, 1998; Colby \& Goldberg, 1999; Heide et al., 2001; Tobler et al., 2001; Medendorp et al., 2003).

Within this context, it is as yet unclear whether the updating process in the PPC is more related to perceptual localization (Colby \& Goldberg, 1999) or to motor intention (Bracewell et al., 1996; Mazzoni et al., 1996). Recently, event-related potentials (ERPs) correlating with updating in a double-step task have been identified over parietal areas (Bellebaum et al., 2005b). The timing of updating-related parietal activity, starting between 150 and $200 \mathrm{~ms}$ after saccade onset, is more consistent with processes associated with the execution of the second saccade. Asymmetries of parietal ERP sources for leftward and rightward first saccades suggested that the ERP updating effect also included information about the first saccade. Although it was assumed that parietal ERP updating effects originated from corollary discharge signals of the neuronal motor command, the data of this study did not provide direct evidence for this hypothesis.

The present study aimed to explore to what extent subcortical corollary discharge signals related to saccadic eye movements contribute to parietal updating-related ERP effects. Another objective of the study was to elucidate whether thalamic lesions in humans, which lead to an impairment in using corollary discharge information as revealed by an earlier study (Bellebaum et al., 2005a), also affect updating-related cortical processing. 


\section{Materials and methods}

\section{Subjects}

Four patients, two women and two men, with focal ischaemic thalamic lesions and 12 control subjects took part in the experiment. The experiment was undertaken with the understanding and written consent of the subjects. The study conformed to the Declaration of Helsinki and was approved by the Ethics Committee of the RuhrUniversity of Bochum. All procedures were carried out with adequate understanding and written consent of the subjects.

The patients were outpatients of the Klinikum Dortmund, Germany. They had already participated in a previous study which was carried out 18 months before the present study (Bellebaum et al., 2005a). In the following, the patients will be referred to as Patients 1-4 (Patients $3,11,8$ and 4 in the original article). Thalamic lesions were verified by magnetic resonance imaging in transverse and coronal sections obtained at a neurological follow-up examination approximately 30 months before the present study. Affected thalamic nuclei were determined using an established atlas (Mai et al., 1997). Figure 1A shows magnetic resonance images of lesion locations. In the previous study, saccade accuracy in a double-step task was assessed [for results see Fig. 1B; Fig. 1 was modified with permission from Oxford University Press (Bellebaum et al., 2005a)]. The double-step task requires the use of corollary discharge information (for details about the task see below). In all four patients, unilateral deficits in using corollary discharge information were revealed by asymmetries in performance (see Fig. 1B).

Patient 1 was a right-handed, 26-year-old woman. She had a lesion in the left medial thalamus, affecting MD and the centromedianparafascicular complex (see Fig. 1A). At neurological follow-up there were no neurological deficits. Assessment of saccade accuracy had revealed a unilateral impairment in using corollary discharge information ipsilateral to the side of lesion (Fig. 1B). The lesion-test interval for Patient 1 was 106 months.

Patient 2, a 60-year-old right-handed woman, had a selective lesion in nucleus VL of the left thalamus (Fig. 1A). The neurological followup examination revealed a mild somatosensory deficit in the right hand. In contrast to Patient 1 , she had a contralateral impairment in using corollary discharge information (Fig. 1B). The interval between lesion and ERP assessment was 92 months.

Similarly, Patients 3 and 4 (both men and right-handed, 72 and 48 years old, respectively) had left-sided lesions affecting VL (Fig. 1A) and showed asymmetries in saccade performance, with a deficit in using corollary discharge information contralateral to the side of lesion (Fig. 1B). The times after the lesion were 96 and 77 months, respectively. In Patient 3, there were no neurological deficits, whereas Patient 4 had mild somatosensory problems in the right hand.

Healthy control subjects, seven women and five men, were recruited from a pool of volunteers at the Department of Neuropsychology at the Institute of Cognitive Neuroscience of the Ruhr-University Bochum. Mean age was 50.25 years $(\mathrm{SD}=14.96$, range 27 68 years). Eleven control subjects were right-handed and one was left-handed. All subjects had normal or corrected-to-normal vision.

\section{Stimuli and task}

In the experimental and control conditions (CC), subjects performed a saccadic double-step task, i.e. they had to perform two successive saccades to flashed targets. The experimental condition was identical to the task which yielded impairments in thalamic lesion patients. The stimulus timings in the $\mathrm{CC}$ were shorter than those of the earlier study, to achieve similar saccade latencies in both conditions (Bellebaum et al., 2005a,b).

At the beginning of each trial, a central fixation point appeared on the computer screen. After an unpredictable delay (1200-1800 ms), the fixation point disappeared and the first saccade target came on. It was located at either $7.5^{\circ}$ visual angle to the left or right of the fixation point. Thereafter the second target was presented on the screen, $7.5^{\circ}$ visual angle upward or downward from the first target. Subjects thus had to first perform a horizontal saccade and then a vertical saccade. The presentation times of the first and second target were 120 and $50 \mathrm{~ms}$, respectively. All visual stimuli were red dots of $0.5^{\circ}$ visual angle diameter.

The only difference between the conditions was the relative timing of the target stimuli. In the experimental condition, both target stimuli were presented before onset of the first saccade, so that retino-spatial dissonance was induced. The retinal vector, under which the second target was seen, was different from the movement vector for reaching the second target. Therefore, this condition will be referred to as the retino-spatial dissonance condition (RDC). In the $\mathrm{CC}$ there was a delay between the offset of the first and the onset of the second target (see Fig. 2).

The delay was introduced to ascertain that subjects performed the first saccade before the second target was presented, i.e. there was no retino-spatial dissonance. As saccade latency increases with increasing age, the between-stimulus interval was varied depending on the age of the subject. For subjects under 40 years of age, i.e. for Patient 1 and for four control subjects, the interval was $250 \mathrm{~ms}$ whereas for the other subjects it was $350 \mathrm{~ms}$. In Patient 2, the interval was changed from 350 to $550 \mathrm{~ms}$ after one third of the trials because of very long saccade latencies.

A fixation condition served to control for the effects of visual stimulation on RDC trials. The visual stimulation was exactly the same as on RDC trials but subjects were asked to keep their eyes on the location of the central fixation point, even when it was turned off and visual stimuli were presented laterally. To ascertain that subjects were still attending to the target stimuli, they were asked to press one of two buttons when the location of the second target was presented above the location of the first target and to press the other button when it was below the first target.

On CC trials, the second target stimulus appeared after the first saccade was executed. To control for visual stimulation on CC trials, all patients and eight control subjects performed a one-saccade condition, in which subjects were instructed to perform only one saccade to the location of the first target. While they were keeping their eyes on the location of the first target, a second dot was presented above or below the first target. Similar to the interstimulus interval on $\mathrm{CC}$ trials, the delay in the one-saccade condition was varied depending on the age of the subject. The delay was $400 \mathrm{~ms}$ for Patient 1. In Patients 2 and 3, the delay was changed after the first half of the trials because both showed longer latencies than expected (450-550 $\mathrm{ms}$ in Patient 2 and 400-500 ms in Patient 3). For Patient 4 and the eight control subjects, who performed the one-saccade condition, the interstimulus interval was $500 \mathrm{~ms}$.

In four control subjects, data from $\mathrm{CC}$ trials were used to obtain visual evoked potentials (VEPs) in response to the second target stimulus. For this purpose, only those trials were used in which the first saccade was already completed when the second target appeared and the second saccade was executed at least $300 \mathrm{~ms}$ after the presentation of the second target stimulus.

All subjects performed three blocks of RDC and CC trials. In addition, the patients and eight control subjects performed two blocks of the fixation and one-saccade conditions; four control subjects 


\section{A}

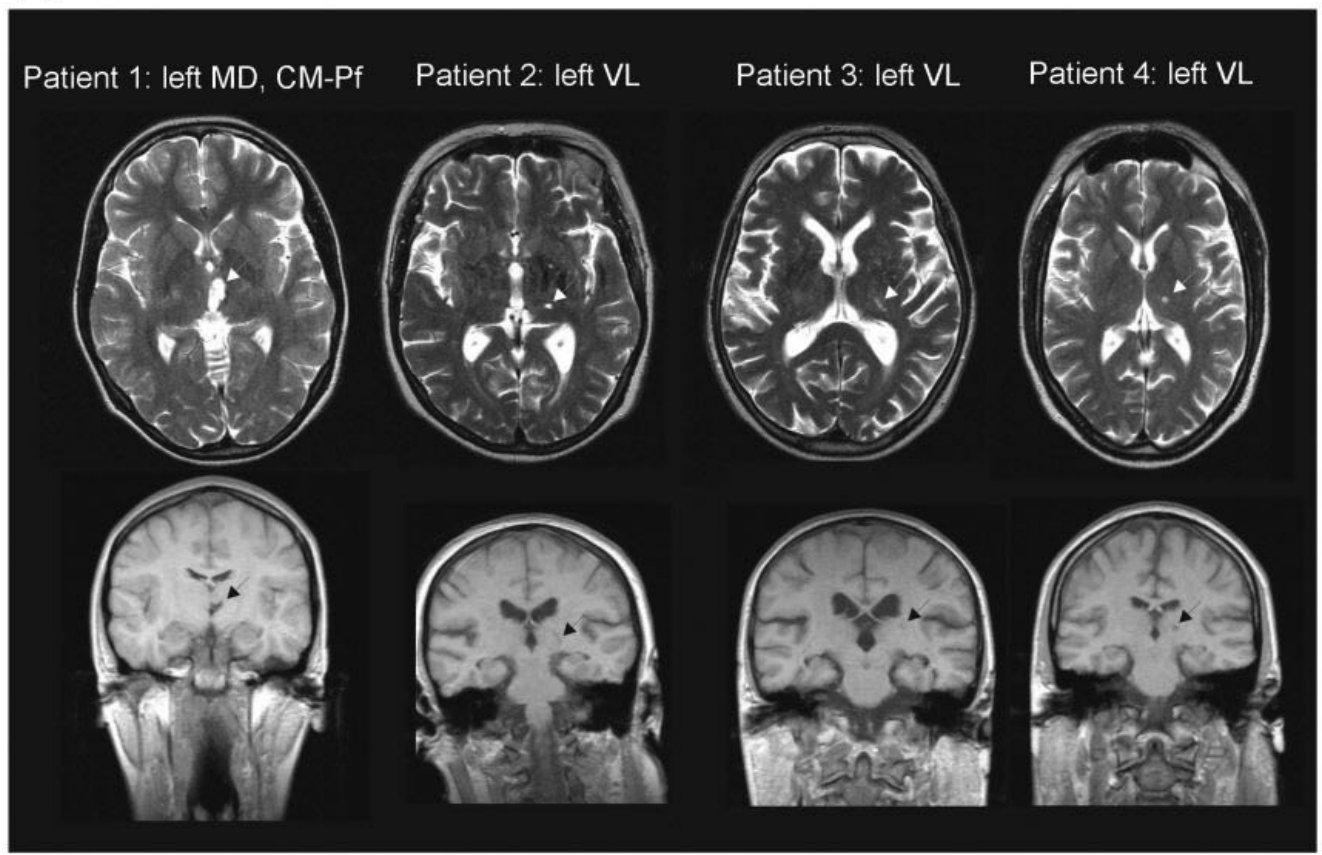

B

— experimental condition: corollary discharge required

— control condition: no corollary discharge required

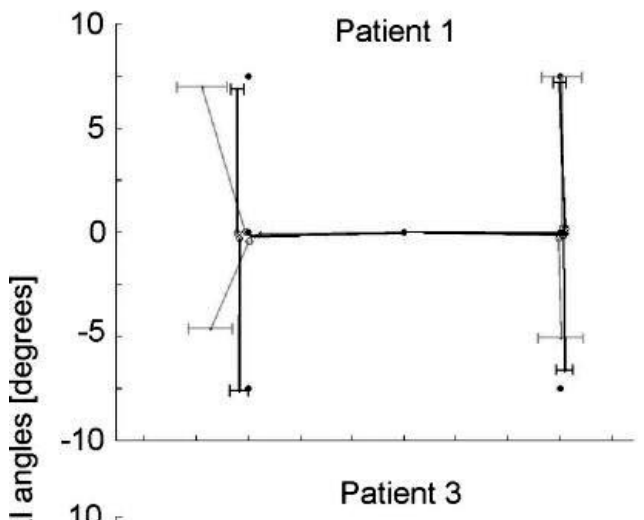

Patient 2
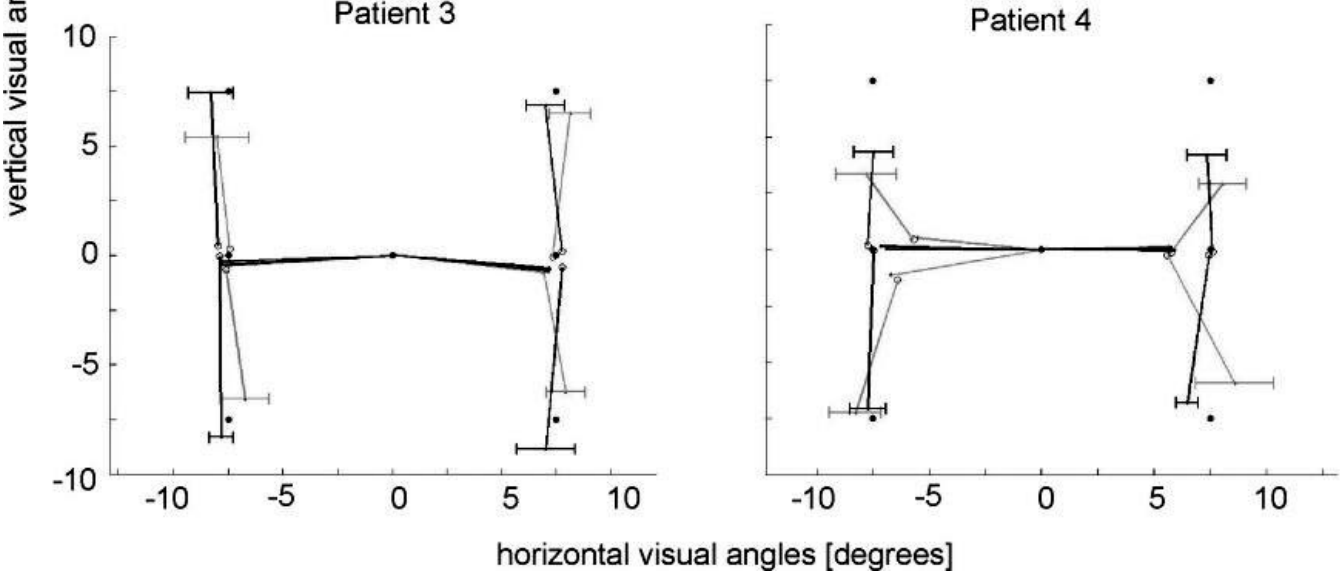

FIG. 1. (A) Transverse and coronal magnetic resonance images of lesion locations. White and black arrows indicate lesion locations. (B) Medians of saccade directions and amplitudes in the saccadic double-step task. Subjects were required to perform two successive saccades, from the fixation point in the middle to the first target stimulus (left or right) and from there to the second target stimulus (up or down). Data were recorded in an extra session approximately 18 months before event-related potentials were assessed. All patients showed significantly enlarged asymmetries in performance. The saccade accuracy difference between trials with and without the requirement to use corollary discharge information was very different for trials with leftward and rightward first saccades. Modified with permission from Oxford University Press (Bellebaum et al., 2005a). Patients 1-4 correspond to patients 3, 11, 8 and 4 in the original publication. CM-Pf, centromedian-parafascicular complex; MD, medio-dorsal nucleus of the thalamus; VL, ventrolateral nucleus of the thalamus. 
A

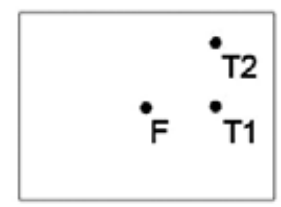

B

experimental condition (RDC)

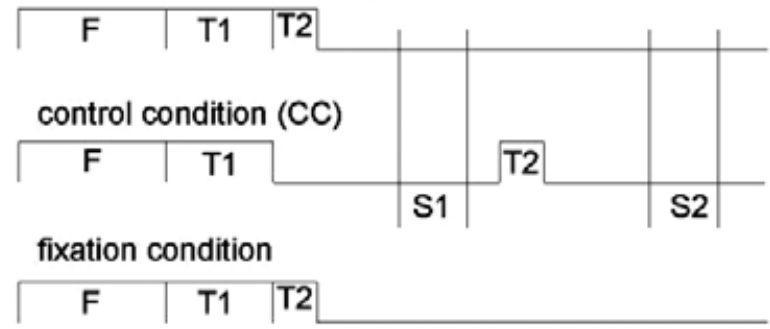

\section{one-saccade condition}

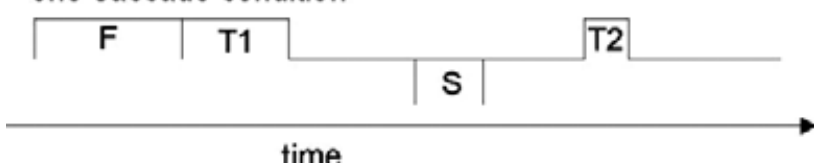

time

FIG. 2. (A) Locations of fixation point and target stimuli in a given trial. (B) Sequence of stimuli and saccades in four conditions. In the experimental condition both targets were flashed in succession, so that they had already disappeared before the first saccade was executed. In the control condition (CC) there was a delay between the presentations of targets 1 and 2, in which the first saccade could be executed. In the fixation condition no saccade was required. Visual stimulation was exactly the same as in the experimental condition. Similar to the CC, a delay between the presentations of both target stimuli was introduced in the one-saccade condition. A second saccade was not required. F, fixation point; $\mathrm{T}$, target; $\mathrm{S}$, saccade; RDC, retino-spatial dissonance condition.

performed three blocks of the fixation condition. Each block consisted of 60 trials. Trials with different target locations were presented in random order.

\section{Data recording}

Throughout the experiment, electroencephalograms were recorded from 30 scalp sites according to the International 10-20 System (F7, F3, Fz, F4, F8, FT7, FC3, FCz, FC4, FT8, T7, C3, Cz, C4, T8, TP7, $\mathrm{CP} 3, \mathrm{CPz}, \mathrm{CP} 4, \mathrm{TP} 8, \mathrm{P} 7, \mathrm{P} 3, \mathrm{Pz}, \mathrm{P} 4, \mathrm{P} 8, \mathrm{PO} 7, \mathrm{PO} 3, \mathrm{POz}, \mathrm{PO} 4$ and $\mathrm{PO} 8)$, referenced to linked mastoids. Silver-silver chloride electrodes fixed in an elastic cap were used.

Subjects were seated $57 \mathrm{~cm}$ in front of a computer monitor (LCD display) on which the visual stimuli were presented. A chin rest was used to stabilize the head position. The experiment was conducted in the dark. The EOG was recorded from electrodes at the outer canthi of both eyes, and above and below the left eye to determine the onset of horizontal and vertical eye movements. A Neuroscan Synamps System (Compumedics, El Paso, TX, USA) and the appropriate software were used for recording. All data were sampled at a rate of $500 \mathrm{~Hz}$. The impedance was kept below $5 \mathrm{k} \Omega$.

\section{Procedure}

Subjects were told that the study aimed to assess brain activity related to eye movements. For the conditions which required two successive eye movements, participants were instructed that they would see a central fixation point on the screen, followed by a first and second target stimulus. They were asked to make two successive eye movements to the target positions as fast and as accurately as possible. For the fixation condition, they were asked to look at the position of the central fixation point during the whole block of trials. They would see two successive stimuli flashed on the screen. They were asked to attend to the target stimuli and to press a response button when the second target stimulus appeared above the first target stimulus and to press another button when it appeared below.

In the one-saccade condition, subjects were asked to perform a saccade to the location of the first target as fast and as accurately as possible and to keep fixating the location of the first target while the second target was presented.

\section{Analysis of electroencephalography and saccade data}

Data were analysed off-line. The initial analysis steps were performed using the BRAIN VISION ANALYSER software package (BrainProducts, München, Germany). Raw data were filtered with a $0.1-\mathrm{Hz}$ high-pass and a $40-\mathrm{Hz}$ low-pass filter. Segments were then created, ranging from $600 \mathrm{~ms}$ before to $1500 \mathrm{~ms}$ after onset of the first target. After ocular artefacts had been corrected using the method described by Gratton et al. (1983), segments were baseline-corrected relative to the average signal in the first $400 \mathrm{~ms}$ of the time epoch, i.e. from 600 to $200 \mathrm{~ms}$ before first target onset.

The raw data of all 60 trials per block were then exported and further analysed using MATLAB (The Mathworks, Natick, Massachusetts, USA). As we did not aim to assess saccade amplitude by EOG, the signal was not calibrated. Before recording started, visual inspection of five to 10 test trials ascertained that horizontal and vertical saccades resulted in easily identifiable deflections of the horizontal and vertical EOG, respectively. In accordance with the procedure of an earlier study (Bellebaum et al., 2005b), a saccade was scored if the horizontal or vertical EOG signal increased or decreased in 10 or more consecutive time frames (epoch of $20 \mathrm{~ms}$ ) and if the amplitude of the signal changed by more than $40 \mu \mathrm{V}$ during this interval. If the signal returned to baseline at a similar rate, it was considered a blink rather than a saccade.

Only RDC and CC trials with two successive saccades entered final analysis. In addition, the timing of the saccades was considered. Only those RDC trials were included in which retino-spatial dissonance was involved, i.e. the first saccade had to start after the second target had already disappeared. In the $\mathrm{CC}$, only those trials entered analysis in which the first saccade was already executed when the second target was presented, i.e. the trials did not involve retino-spatial dissonance. For both conditions, the minimum interval between first and second saccade onset was $200 \mathrm{~ms}$. Trials with artefacts were rejected automatically. Segments with a difference between the highest and lowest data point exceeding $150 \mu \mathrm{V}$ were excluded.

In the next analysis step, every segment fulfilling the criteria outlined above was realigned with respect to the onset of the first saccade, with every new ERP segment starting $400 \mathrm{~ms}$ before and ending $500 \mathrm{~ms}$ after saccade onset. ERPs of all remaining trials per subject and condition were then averaged separately for leftward and rightward trials, i.e. trials with leftward and rightward first saccades. Data for trials with upward or downward second saccades were pooled.

In the fixation condition, only trials without saccades entered analysis. After removal of artefact trials, ERPs time-locked to first target onset were averaged for every subject, separately for trials with left and right stimuli. 
Trials of the one-saccade condition were aligned to second target onset. Artefact-free trials entered analysis if one saccade was performed per trial and if saccade offset occurred not later than $100 \mathrm{~ms}$ before the presentation of the second target. All remaining trials were averaged per subject.

\section{Statistical analysis}

The ERP data of control subjects were analysed first. Nine electrodes were chosen for analysis (FC3, FCz, FC4, CP3, CPz, CP4, PO3, POz and PO4). The results obtained at these electrode sites reflect the general ERP pattern. Exploratory analyses with a different subset of electrodes (F3, Fz, F4, C3, Cz, C4, P3, Pz and P4) revealed the same general pattern of results. To determine times of significant amplitude differences between conditions, a previously described method was used (Rugg et al., 1995).

\section{Results}

\section{Number of trials entering analysis}

For control subjects, the average number of trials entering analysis was 67 (SD 27) for leftward RDC trials and 65 (SD 26) for rightward RDC trials, whereas for leftward and rightward CC trials 71 (SD 26) and 73 (SD 27) trials entered analysis. For the patients, the average numbers of trials entering analysis were 43 (SD 20) and 42 (SD 17) for leftward and rightward RDC trials. On CC trials, on average 49 leftward and 49 rightward trials entered analysis (SD 13 and SD 14, respectively). No significant main effects or interactions emerged (all $P>0.08)$, as revealed by an ANOVA with the within-subjects factors CONDITION (RDC vs. CC) and DIRECTION (leftward vs. rightward) and the between-subjects factor GROUP.

\section{Inter-saccade intervals}

As ERPs were time-locked to first saccade onset, the interval between first and second saccade onset is of particular interest in the present study. Figure 3 shows group data of the intersaccade interval as well as data of individual patients in leftward and rightward RDC and CC trials. The data refer to those trials that fulfilled the timing criteria and thus entered ERP analysis (see Materials and methods). An ANOVA with the factor GROUP (patients vs. controls), and the within-subjects factors CONDITION (RDC vs. CC) and DIRECTION (leftward vs. rightward) did not yield significant main effects or interactions (all $P>0.12$ ).

\section{Saccade-locked event-related potentials in control subjects}

Figure 4A shows grand-average ERPs of control subjects at nine electrode sites for RDC and CC trials. Similar to the results described for younger subjects in a previous study (Bellebaum et al., 2005b), the amplitude is more positive on RDC compared with CC trials in a time window starting about $150 \mathrm{~ms}$ after first saccade onset in both leftward and rightward trials. The larger positivity in RDC compared with CC trials was related to updating of visual space (Bellebaum et al., 2005b) and will therefore be referred to as updating effect in the following.

To determine time windows with a significant updating effect in the control subjects of the present study, point-by-point $t$-tests (i.e. every $2 \mathrm{~ms}$ ) were performed for the RDC-CC difference for each electrode. The conditions were considered to differ significantly when at least 15 consecutive $t$-tests were significant at the 0.05 level (Rugg et al., 1995). The first of the 15 or more time points was considered the onset of the updating effect.

Horizontal bars above ERPs in Fig. 4A indicate times of significant amplitude difference. The updating effect starts to emerge on average at about $150 \mathrm{~ms}$ after first saccade onset for both leftward and rightward trials. At frontocentral and centroparietal electrode sites it starts earlier (between 130 and $140 \mathrm{~ms}$ ) than at parieto-occipital sites (between 160 and $170 \mathrm{~ms}$ ).

Topographic activity maps of the difference waves (RDC-CC trials) in healthy control subjects between 200 and $400 \mathrm{~ms}$ after first saccade onset replicated the laterality effect described in an earlier study (Bellebaum et al., 2005b). In leftward trials, relative positivity is larger
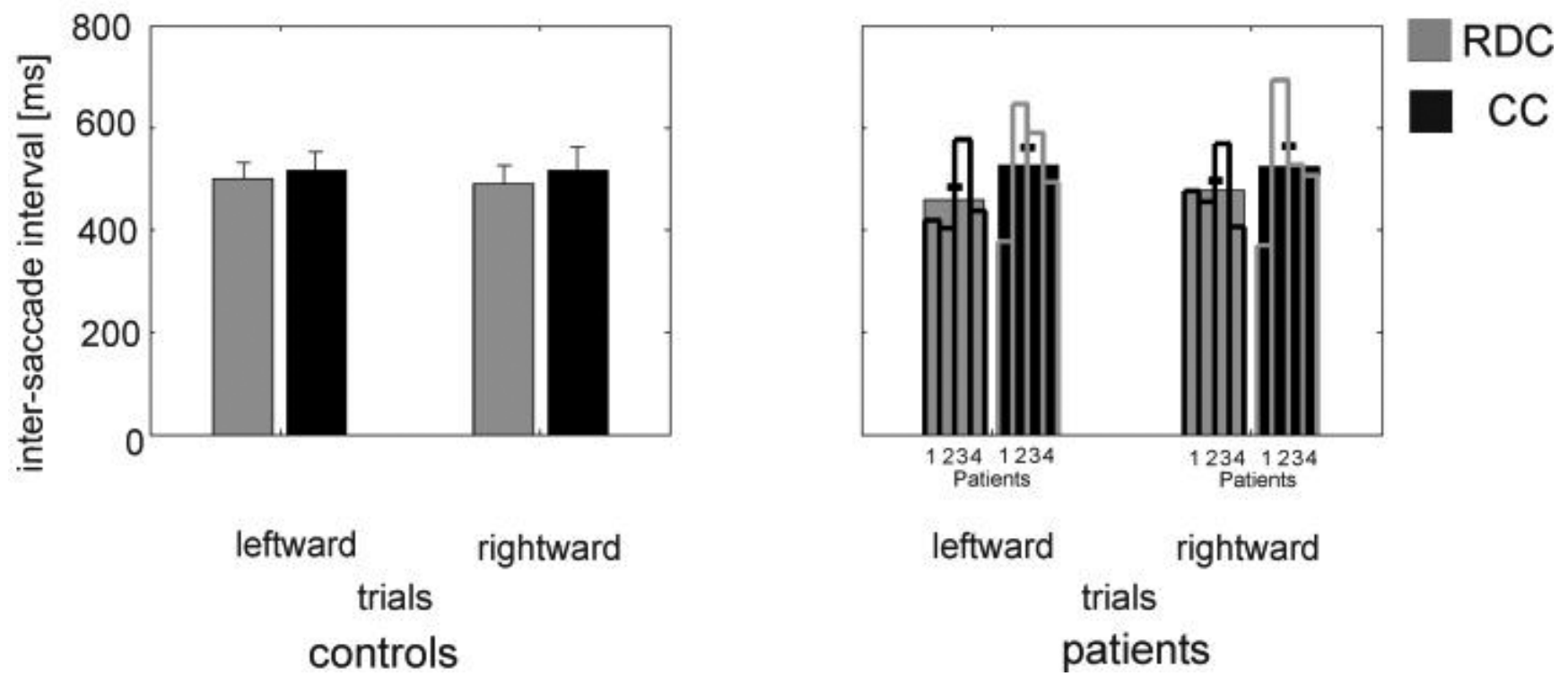

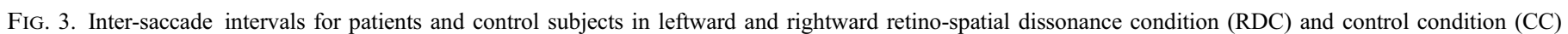
trials. Intervals are illustrated for the patient and control groups as well as for individual patients. 


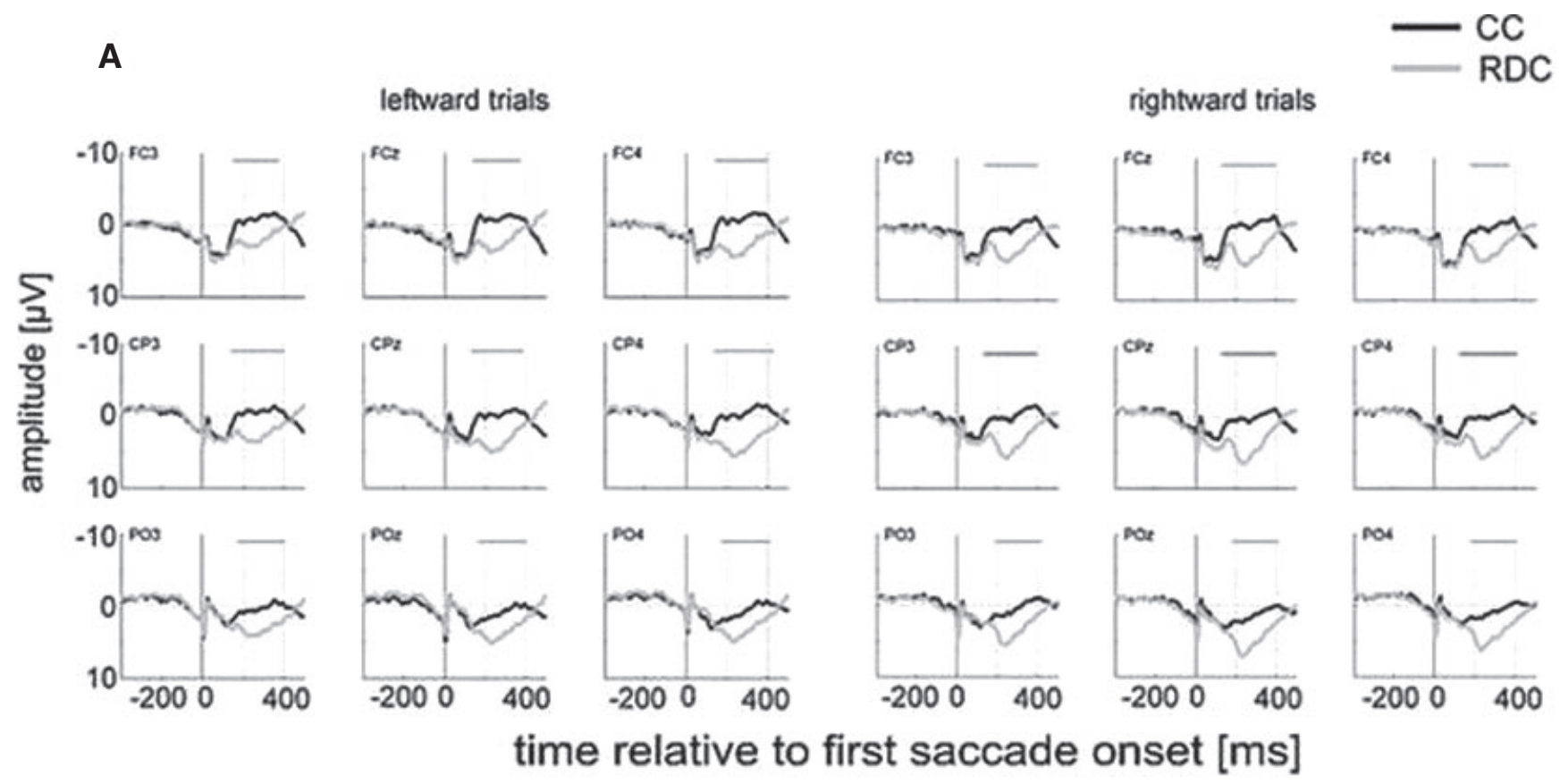

B
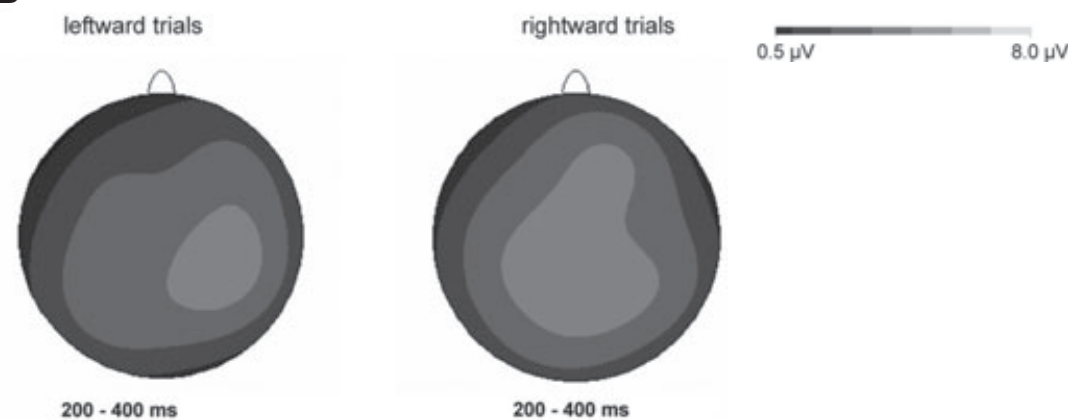

FIG. 4. (A) Control subjects' grand-average event-related potentials (ERPs) for leftward and rightward retino-spatial dissonance condition (RDC) (in grey) and control condition (CC) (in black) trials. ERPs are aligned to first saccade onset. Black bars above the traces indicate times of significant difference between conditions. (B) Topographic maps of control subjects' ERP difference waves (RDC-CC trials) for leftward and rightward trials between 200 and 400 ms after first saccade onset.

over the right hemisphere, especially over the parietal cortex. In contrast, both hemispheres appear to be involved in rightward trials (Fig. 4B).

\section{Effect of VEPS}

As the timing of the second target stimulus was different in RDC and CC trials, it might be argued that VEPs caused by the presentation of the second target stimulus contributed to the updating effects described above. To control for VEP effects caused by target presentation on saccade-triggered ERPs in RDC and CC trials, VEPs derived from the fixation and one-saccade conditions were used. In four control subjects, who did not perform the one-saccade condition, VEPs were obtained from CC trials, in which the second saccade occurred very late (see Materials and methods). As there were large interindividual differences with respect to VEP amplitudes in the fixation and one-saccade conditions and as VEPs were not clearly seen in all subjects, an exploratory analysis on the effect of VEPs was performed in one control subject, to illustrate the potential effects of
VEPs on saccade-locked ERPs. This procedure was modelled on a previous study (Thickbroom \& Mastaglia, 1985).

Figure 5A and B shows average VEPs at two electrode sites for the fixation and one-saccade conditions in one control subject with particularly large VEPs. In the fixation condition, two distinct responses to the two target stimuli can be observed at about 220 and $360 \mathrm{~ms}$. In the one-saccade condition, traces are time-locked to second target onset. Therefore, only one peak at $220 \mathrm{~ms}$ occurs in response to the second target.

To remove effects of visual stimulation from saccade-locked ERPs, only VEPs in response to the second stimulus in the fixation and onesaccade conditions were used. A repeated sum of the mean VEP from the fixation condition was formed, dispersed according to the histogram of the timing of the second target stimulus in relation to first saccade onset on RDC trials (Thickbroom \& Mastaglia, 1985). Assuming that VEPs contribute linearly to ERPs, these summed VEPs were subtracted from saccade-locked ERPs of RDC trials.

Similarly, a repeated sum of temporally dispersed VEPs obtained from the one-saccade condition was subtracted from saccade-locked ERPs of CC trials. In Fig. 5C the original as well as the corrected 

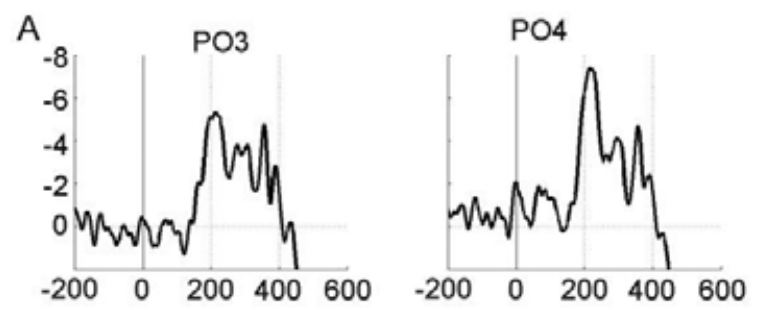

time relative to first stimulus onset [ms]

B

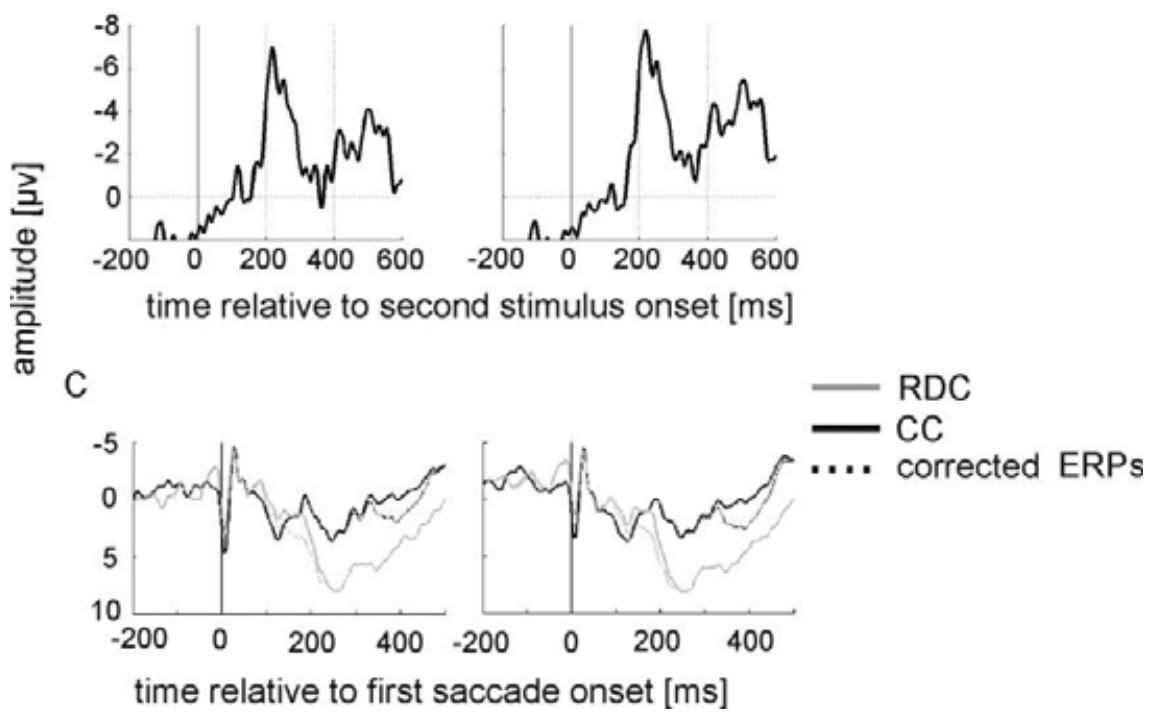

FIG. 5. Visually evoked potentials of one control subject in response to (A) both target stimuli in the fixation condition and (B) the second target stimulus in the one-saccade condition. (C) Original and corrected saccade-locked event-related potentials (ERPs). CC, control condition; RDC, retino-spatial dissonance condition.

ERPs are shown. Although the subject had large-amplitude VEPs to target stimuli, the effect of VEPs on saccade-locked ERPs is minimal. In accordance with earlier work (Thickbroom \& Mastaglia, 1985), saccade-locked ERPs did not change substantially when temporally dispersed VEPs elicited by visual target stimuli were subtracted. This finding as well as the fact that control subjects' saccade-locked ERPs in the present study were similar to the ERPs obtained in an earlier study, in which corrected ERPs were used (Bellebaum et al., 2005b), led us to assume that visual effects on saccade-locked ERPs were minimal. Therefore, uncorrected saccade-locked ERPs entered analysis.

\section{Saccade-locked event-related potentials of individual patients}

In Fig. 6 saccade-locked ERPs of all four patients are shown for nine electrode sites, separately for leftward and rightward trials.

Z-scores were computed for the size of the updating effect in each individual patient, relative to control subjects at nine electrode sites. The size of the updating effect for a given electrode was computed as the mean amplitude difference between RDC and CC trials in the time range in which both conditions differed significantly in control subjects (see bars above ERPs in Fig. 4A). Z-scores below -1.64 $(P<0.05)$ were considered to reflect a significant reduction of the size of the updating effect.

For leftward trials, Patient 1 (left-sided MD and centromedianparafascicular complex lesion, ipsilateral impairment) showed a significantly reduced updating effect at $\mathrm{FC} 3(\mathrm{Z}=-2.39)$. Z-scores for the other electrode sites were all larger than -1.39 .

Of the other patients, who all had left-sided VL lesions and contralateral impairments, only Patient 2 showed significantly smaller updating effects than controls in leftward trials. Reductions were found over parietal cortex (Z-scores of -2.52 and -2.27 at electrode sites CP4 and PO4). The Z-scores at CP3 and PO3 approached significance $(Z=-1.51$ and $Z=-1.61$, respectively). The updating effects in Patients 3 and 4 were in the normal range (all Z-scores $>0.26$ ).

For rightward trials, Patient 1 showed intact updating effects (all Z-scores $>-1.32$ ). For the other patients, the pattern was similar to the pattern in leftward trials, with Patient 2 showing reduced effects at parietal sites (Z-scores of $-1.68,-1.91$ and -1.71 at electrode sites $\mathrm{CP} 4, \mathrm{POz}$ and PO4; $\mathrm{Z}=-1.58$ at PO3), whereas $\mathrm{Z}$-scores in the other patients did not approach significance (all $\mathrm{Z}>-1.27$ ).

As all patients had unilateral lesions and unilateral impairments in updating visual space, one would expect a lateralized reduction of ERP updating effects. Such effects may have been masked by the considerable interindividual variability in control subjects. To further explore potential laterality effects, the intraindividual difference between updating effects for rightward and leftward trials was computed, subtracting the updating effects for leftward trials from those for rightward trials for every electrode site. These asymmetry scores were then Z-standardized relative to control subjects. Large Z-scores $(>1.64)$ indicate an asymmetry with larger updating effects for rightward trials, whereas low Z-scores $(<-1.64)$ indicate larger effects for leftward trials.

Two VL-lesioned patients showed significant asymmetry scores at single electrode sites. Although updating effects were generally small in Patient 2, the asymmetry Z-score was -2.41 at $\mathrm{CPz}$, indicating that at this electrode site updating effects were significantly reduced for rightward compared with leftward trials. At the other sites Z-scores were larger than -1.17 .

Asymmetry scores of Patient 4 were significantly lower than those of control subjects at electrode sites $\mathrm{FCz}$ and $\mathrm{CPz}(\mathrm{Z}=-2.14$ and 


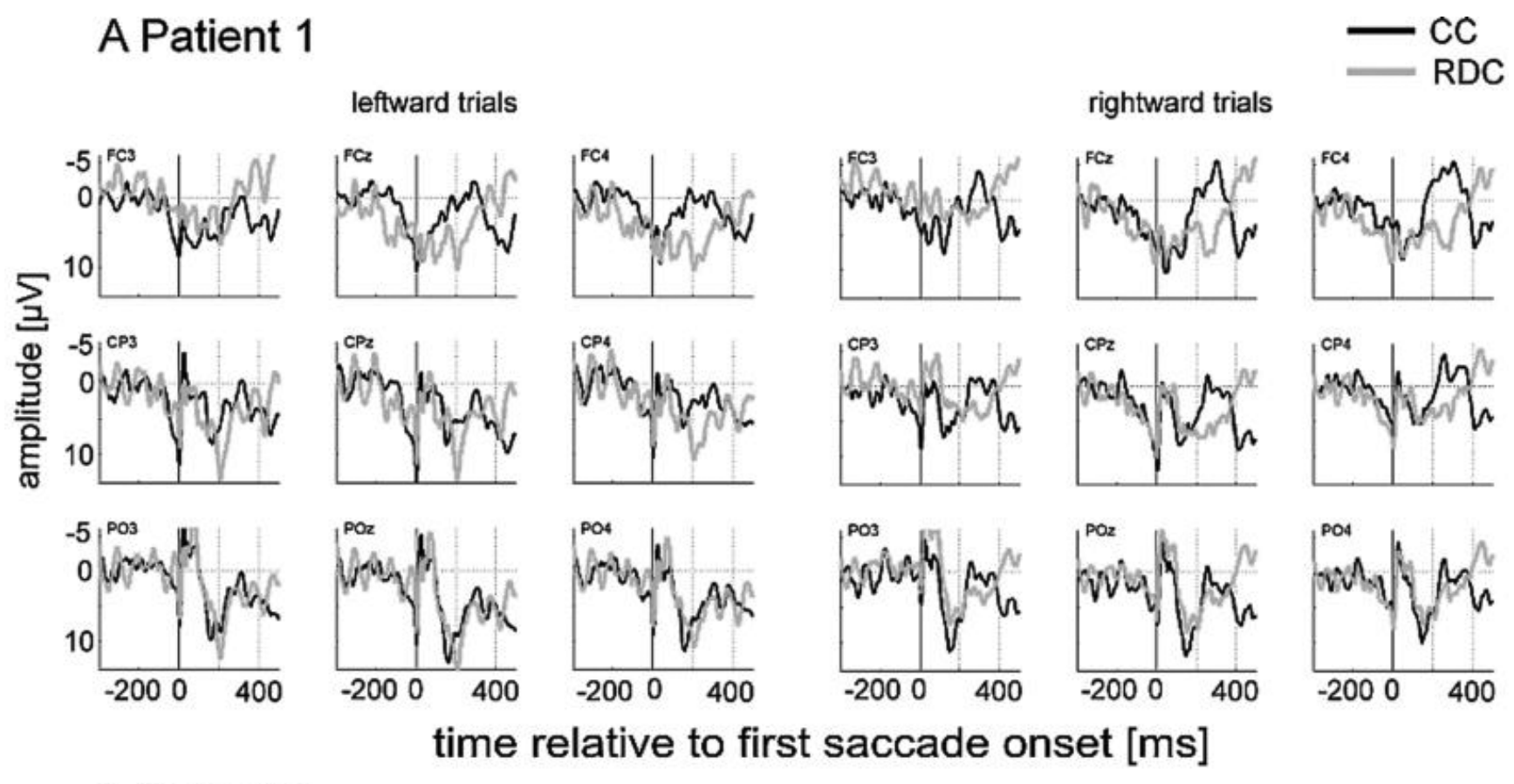

\section{B Patient 2}

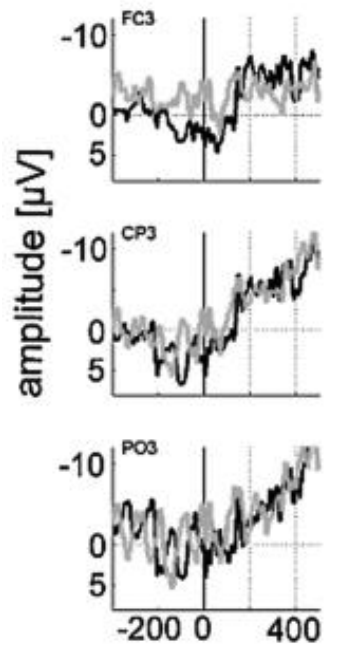

leftward trials
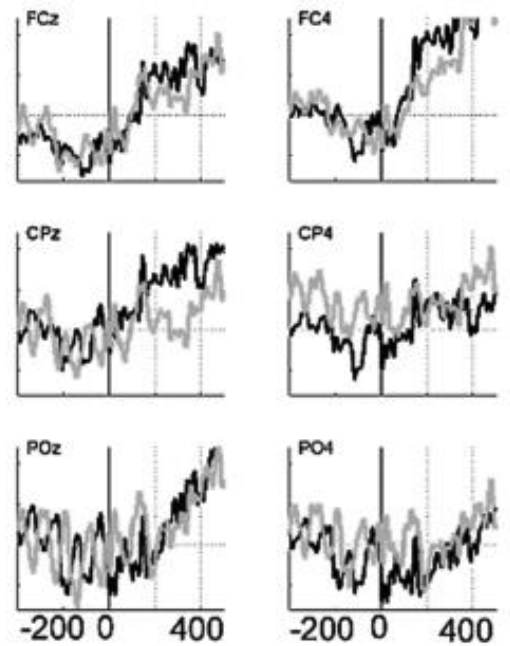

rightward trials
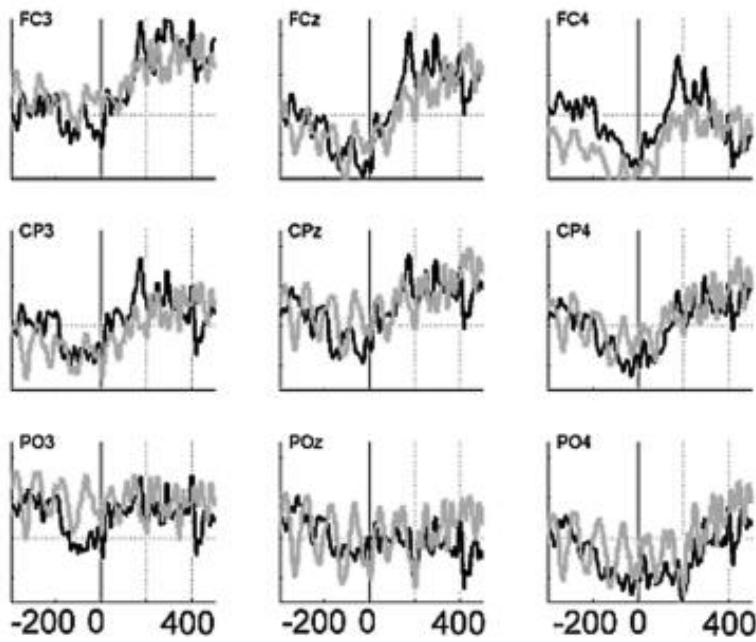

\section{time relative to first saccade onset [ms]}

FIG. 6. Event-related potentials time-locked to first saccade onset in Patients 1-4 (6A-D). CC, control condition; RDC, retino-spatial dissonance condition.

$Z=-2.79$, respectively). At these sites, updating effects were large for leftward trials, whereas they were in the normal range for rightward trials, resulting in a large asymmetry score. At all other electrode sites, asymmetry Z-scores of Patient 4 did not differ from those of controls (all $Z>-1.20$ ).

The results in the other patients were not significant.

\section{Topography of updating effects in patients}

Figure 7 shows the topography of the updating effect (RDC-CC trials) for the four patients. For Patients 2 and 4 the maps reflect the reductions in the size of the updating effect described above.
The maps of Patient 2 clearly differ from those of the average control subjects. In leftward trials, however, a quite normally sized updating effect can be observed near electrode site $\mathrm{CPz}$ and at right frontal sites. In Patient 4, topographic maps of the updating effect clearly show a pronounced updating effect for leftward trials over central cortex and a relative reduction in the size of the updating effect for rightward trials.

Although there is no evidence of reduced updating effects in Patients 1 and 3, there might be a difference in the topography of the updating effect between these patients and the mean topography in the control subjects. In Patient 1, positivity is largest over the left and right frontal cortex for rightward trials, with the centre being over the right hemisphere. In leftward trials the updating effect is generally smaller 


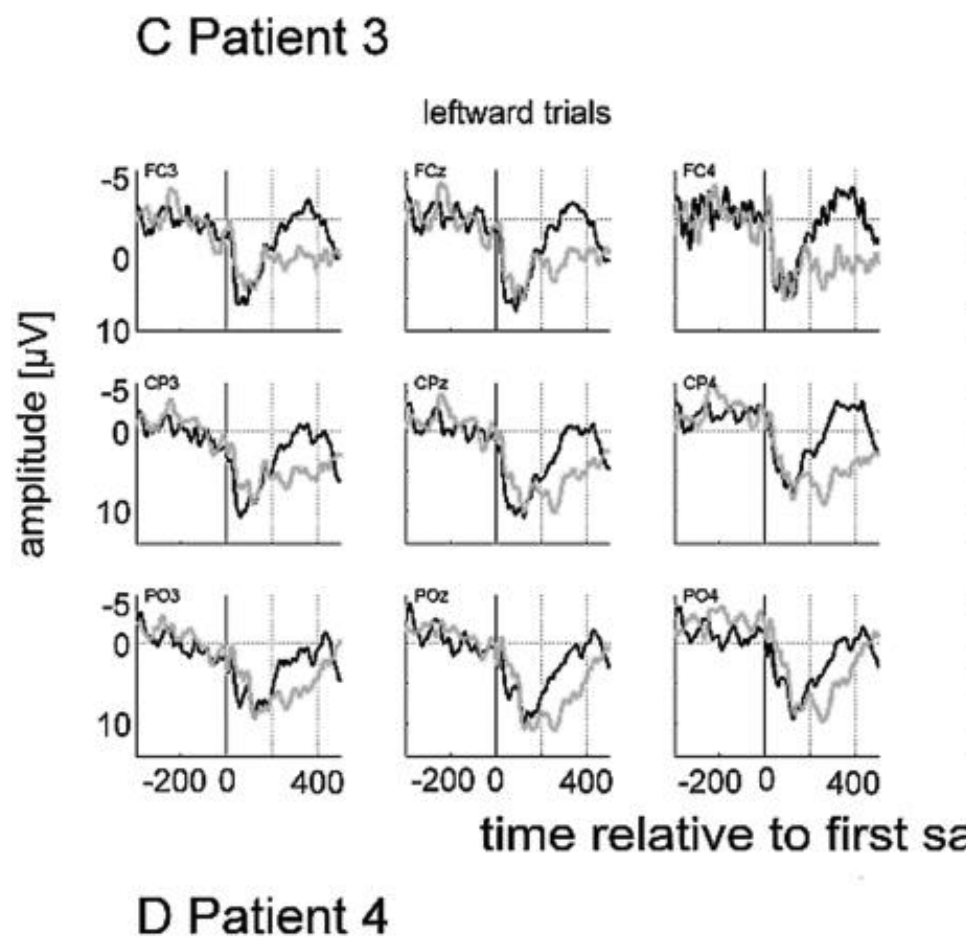

leftward trials
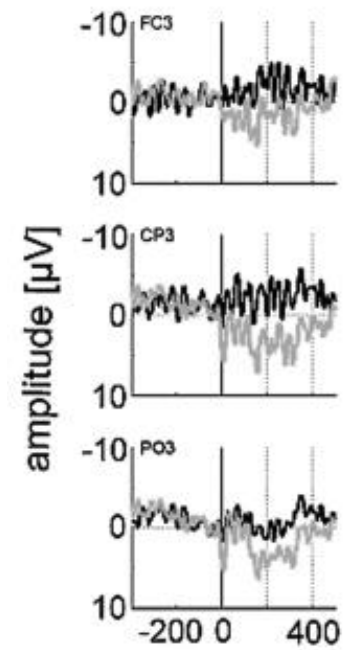
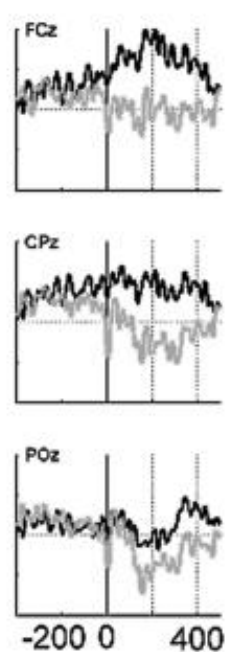

time re
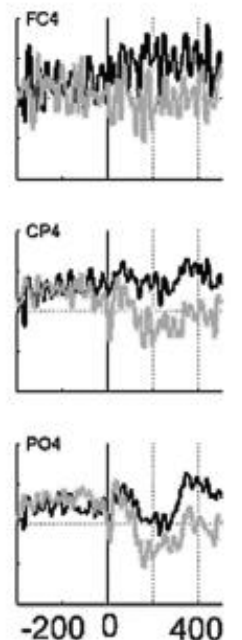
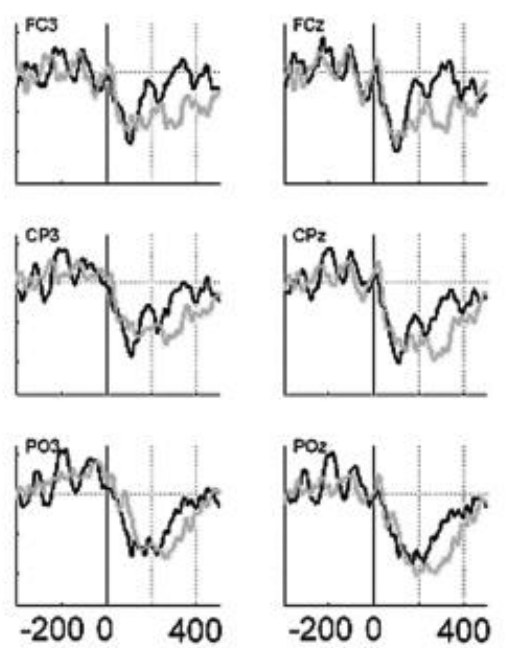
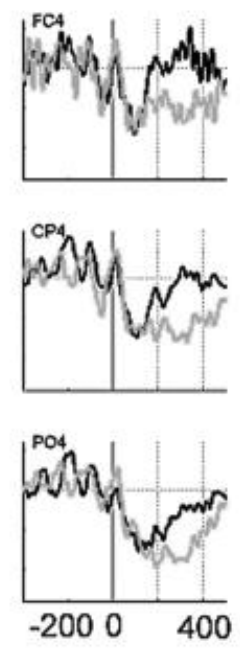
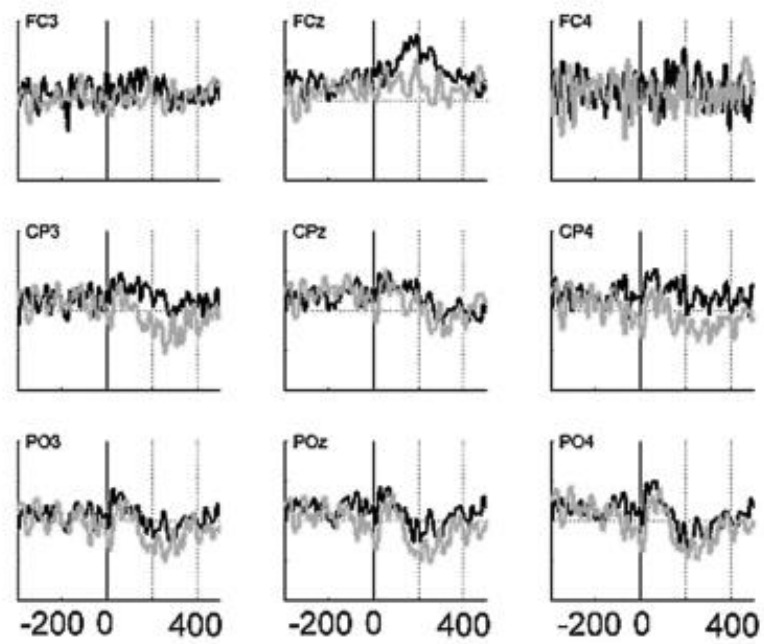

FIG. 6. Continued.

and also more pronounced over the right hemisphere. In Patient 3, the scalp distributions of difference waves are very similar for leftward and rightward trials. As in Patient 1, the effects are largest over the right hemisphere, irrespective of the direction of the first saccade.

To examine whether topographic maps of Patients 1 and 3 reflect significant deviations from the topography of controls, a topographic asymmetry parameter (TOP) was computed for frontocentral, centroparietal and parieto-occipital electrode rows, subtracting the relative size of updating effects at contra- and ipsilateral electrode sites in rightward trials from that in leftward trials

$$
\mathrm{TOP}=(\mathrm{CL}-\mathrm{IL})-(\mathrm{CR}-\mathrm{IR})
$$

where $\mathrm{C}$ is contralateral updating effect, $\mathrm{I}$ is ipsilateral updating effect, $\mathrm{L}$ is leftward trials and $\mathrm{R}$ is rightward trials. For example, the contralateral updating effect for the frontocentral row of electrodes in leftward trials refers to the updating effect at the frontocentral, rightsided electrode, i.e. electrode site FC4. The ipsilateral updating effect in the same row refers to electrode site FC3.

Figure 8 shows asymmetries in the topography of the updating effect for Patients 1 and 3 and for control subjects at frontocentral, centroparietal and parieto-occipital electrode sites in leftward and rightward trials. The diagonal lines indicate similar topographies in leftward and rightward trials, i.e. similar relative involvement of the ipsiand contralateral hemispheres in leftward and rightward trials. After 


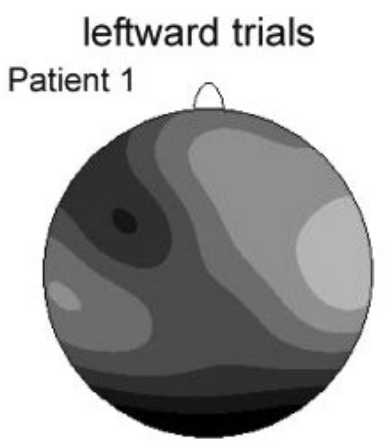

$200-350 \mathrm{~ms}$

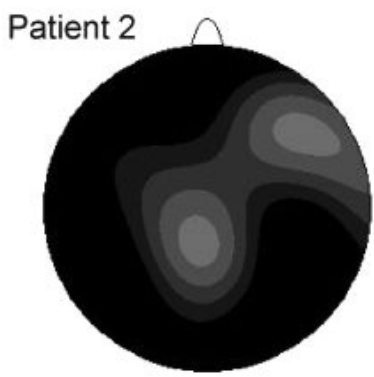

$200-400 \mathrm{~ms}$

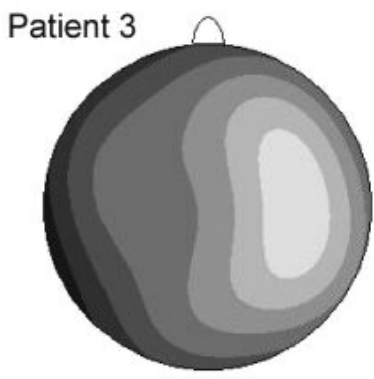

$200-400 \mathrm{~ms}$

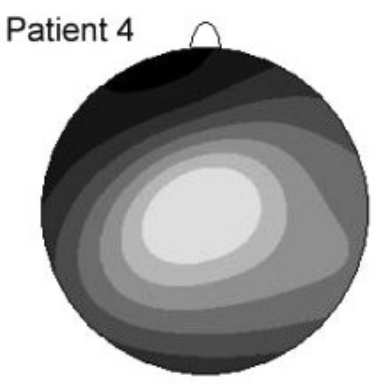

$200-400 \mathrm{~ms}$

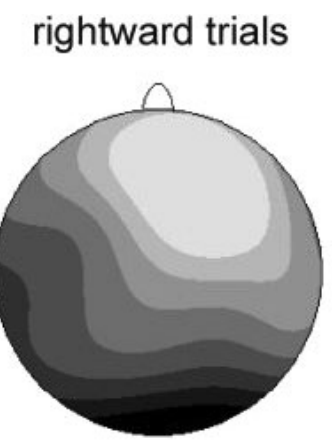

$200-350 \mathrm{~ms}$

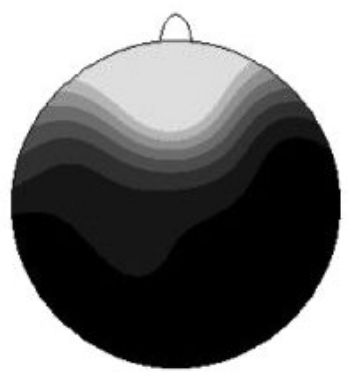

200 - $400 \mathrm{~ms}$

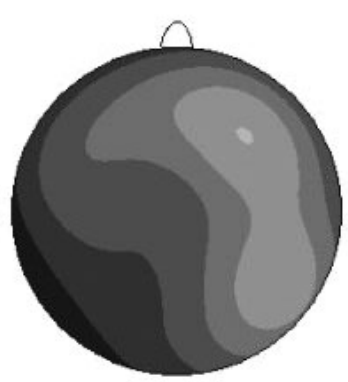

$200-400 \mathrm{~ms}$

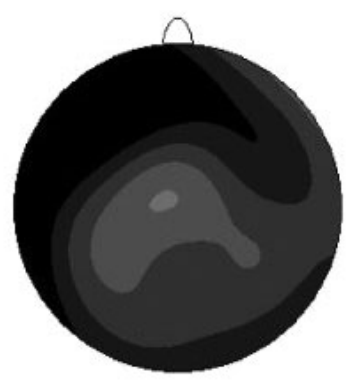

$200-400 \mathrm{~ms}$

\section{$0.5 \mu \mathrm{V}$}

FIG. 7. Individual patients' topographic maps of event-related potential difference waves (retino-spatial dissonance condition-control condition trials) for leftward and rightward trials.

topographic asymmetry parameter scores were computed for all subjects, they were Z-standardized relative to the control subjects' scores.

In Patient 1, the topography Z-score is 4.80 for frontocentral electrodes. For both leftward and rightward trials, the updating effect is larger at electrode site FC4 compared with FC3, suggesting that the right hemisphere is more involved in updating, irrespective of the direction of the first saccade. However, for centroparietal and parietooccipital electrodes, Z-scores in Patient 1 are in the normal range ( $Z=1.10$ and $Z=0.85$, respectively).

The topography of the updating effect in Patient 3 was similar to the topography in Patient 1. Again the right hemisphere seemed to be more involved than the left hemisphere in both rightward and leftward trials. With Z-scores of 2.92 and 2.46, right hemispheric dominance was significant at centroparietal and parieto-occipital electrode sites. At frontal sites, the Z-score was not significantly different from the scores of controls $(Z=1.41)$.

\section{Comparison between updating effects of individual patients and control subjects}

Although small reductions or asymmetries of the size of the updating effect at single electrode sites were also observed in individual control subjects, deviations were always more pronounced in the patients (see Table 1 for a summary of patients' saccade accuracy deficits and ERP patterns). Patient 1 , for example, had a reduced updating effect at only one frontocentral electrode site in leftward trials. However, in addition, the topography of the updating effect was affected. In both leftward and rightward trials the right frontocentral hemisphere was more involved in updating than the left hemisphere. Thus, the pattern in Patient 1 is likely to reflect altered processing.

Patient 2 showed a significantly reduced updating effect at more than one electrode site in both leftward and rightward trials and a significant asymmetry, with the effect being smaller in rightward trials. In none of the control subjects were updating effects reduced for both leftward and rightward trials.

Large asymmetry scores in Patient 4 revealed that the updating effect was reduced for rightward compared with leftward trials. Two control subjects also showed large asymmetry scores but, in contrast to the pattern observed in Patient 4, asymmetries in these subjects do not reflect relative unilateral reductions in the size of the updating effect. In control subject 4, large asymmetry scores are caused by an asymmetry in the timing of the updating effect. For rightward trials, the effect starts at about $50 \mathrm{~ms}$ after first saccade onset, whereas it starts at about $200 \mathrm{~ms}$ in leftward trials. In control subject 11, asymmetry scores are largest for the frontal cortex. They reflect a pronounced laterality of the updating effect, but, nevertheless, for both leftward and rightward trials, the effect is much larger over the contralateral than over the ipsilateral hemisphere.

The size of the updating effect in Patient 3 was in the normal range but, similar to Patient 1, the scalp topography was different from the pattern of normal controls. Over centroparietal and parieto-occipital cortex, the right hemisphere was more involved in the updating process than the left hemisphere, irrespective of the direction of the first saccade. In three control subjects, slightly unusual topography scores were observed but the effects were much larger in Patients 1 and 3. Furthermore, larger involvement of one hemisphere in comparison to the other emerged at two rows of electrodes in Patient 3 (centroparietal and parieto-occipital) instead of only one row in control subjects.

\section{Discussion}

The aim of the present study was to examine the effect of focal thalamic lesions on cortical processing of saccade-related information 
frontocentral

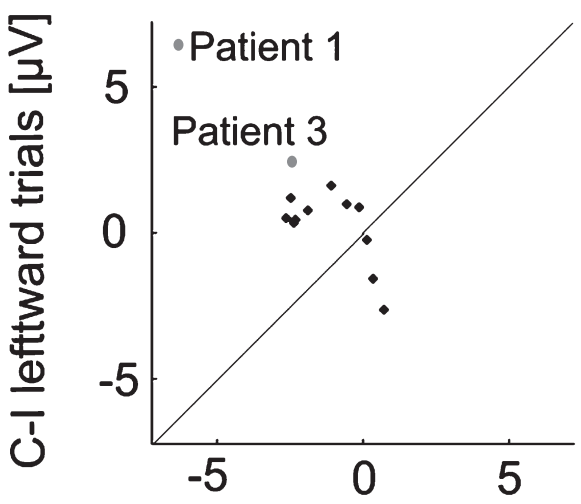

centroparietal

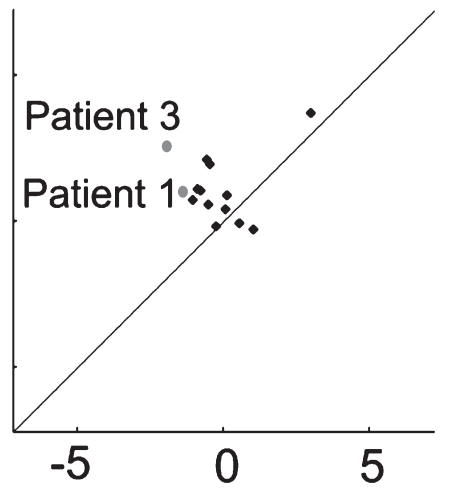

- patients

- controls

parietooccipital

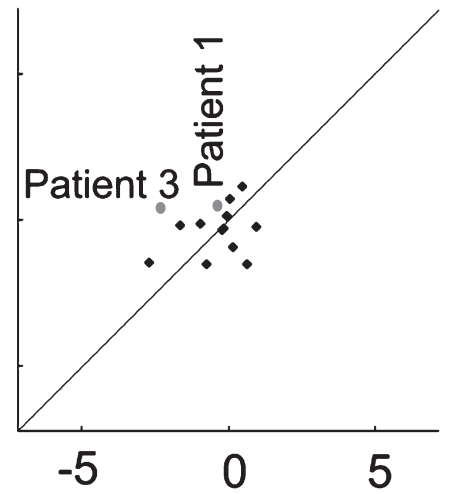

\section{$\mathrm{C}-\mathrm{I}$ rightward trials $[\mu \mathrm{V}]$}

FIG. 8. Differences between contra- and ipsilateral updating effects in leftward and rightward trials for control subjects (black) and two patients (grey). C, contralateral; I, ipsilateral, e.g. the contralateral updating effect in leftward trials for the frontocentral row of electrodes refers to the right-sided frontocentral electrode, i.e. FC4.

TABLE 1. Summary of saccade accuracy deficits and event-related potential (ERP) patterns for all patients

Patient and lesion Saccade accuracy deficit ERP pattern

Patient 1

Left MD/CM-Pf Left-sided

Patient 2

Left VL Right-sided

Patient 3

Left VL Right-sided

Patient 4

Left VL Right-sided
Leftward trials: electrode site FC3, significantly reduced updating effect

Topography: frontocentral electrode sites, larger updating effects over the right hemisphere

Leftward trials: reduced updating effect at electrode sites CP4 and PO4

Rightward trials: reduced updating effect at electrode sites $\mathrm{CP} 4, \mathrm{POz}$ and $\mathrm{PO} 4$

Asymmetry: electrode site $\mathrm{CPz}$, updating effect reduced for rightward trials compared with leftward trials

Topography: centroparietal and parieto-occipital electrode sites: larger updating effects over the right hemisphere

Asymmetry: electrode sites FCZ and CPZ, updating effect reduced for rightward compared with leftward trials

CM-Pf, centromedian-parafascicular complex; MD, medio-dorsal nucleus of the thalamus; VL, ventrolateral nucleus of the thalamus.

in humans. ERPs were recorded while thalamic lesion patients and healthy control subjects performed a saccadic double-step task requiring updating of visual space after the first saccade.

Consistent with a previous study (Bellebaum et al., 2005b), a positive wave over parietal cortex related to updating was observed in a time window starting about $150 \mathrm{~ms}$ after onset of the first saccade in control subjects. In thalamic lesion patients, who were previously shown to be impaired at using corollary discharge information (Bellebaum et al., 2005a), cortical processing of saccade-related information was altered. Despite interindividual differences, the ERP patterns of all four patients show correspondence to their behavioural deficits. Although there was a tendency for fewer valid trials in the patients relative to controls, the ERP differences between control subjects and patients cannot simply be attributed to a reduced number of valid trials in the patients. Comparison of control subjects with a higher and lower number of valid trials did not yield any evidence for differences in the size of the updating effect. The implication of the results for the role of the thalamus in relaying corollary discharge information and for updating-related processing in the PPC will be discussed below.

\section{Human thalamus and corollary discharge}

In previous single case studies, patients with lesions affecting the central or centro-medial thalamus were found to be impaired in tasks requiring the use of corollary discharge information (Gaymard et al., 1994; Versino et al., 2000). A recent group study revealed deficits in patients with lateral thalamic lesions affecting nucleus VL (see Fig. 1, Bellebaum et al., 2005a). Similar to the pattern observed in monkeys following MD lesions (Sommer \& Wurtz, 2002, 2004b), VL-lesioned patients showed impairments in using corollary discharge information when saccades were directed contralaterally to the side of the lesion. The size of the deficit was also similar. In monkeys and humans the deficit amounted to about $20 \%$ of the amplitude of the first saccade in the double-step task. Thus, the trans-thalamic pathway through nucleus MD, which has been described as carrying corollary discharge information by Sommer \& Wurtz (2002, 2004a), may lead through the lateral thalamus in humans. The pronounced deficit observed in Patient 1 (Fig. 1) suggests that nucleus MD might be involved in relaying information about ipsilaterally directed saccades in humans. 


\section{Altered cortical processing related to updating in thalamic lesion patients}

The updating process itself, i.e. the integration of visual and saccaderelated information, has been linked to cortical areas. Lesion and imaging studies provided evidence for a major role of the PPC in updating (Duhamel et al., 1992b; Heide \& Kompf, 1998; Heide et al., 2001; Sereno et al., 2001; Medendorp et al., 2003; Sapir et al., 2004). Evidence from single-cell recordings in the monkey suggests that the FEF, which is reciprocally connected to the PPC (Huerta et al., 1987; Stanton et al., 1995), might also be involved in the updating process (Sommer \& Wurtz, 2002, 2004a,b).

Four patients with unilateral thalamic lesions and unilateral deficits in using corollary discharge information described in a previous study also participated in the present study (Bellebaum et al., 2005a). By showing that updating-related cortical processing in these patients differs from processing in healthy controls, the present study adds further evidence to the notion that the human thalamus conveys corollary discharge information of just-executed saccades to the cortex.

Patients 2, 3 and 4 all had VL lesions and a contralateral impairment in using corollary discharge information. At first sight, their ERP updating effects differed from the effects observed in control subjects in different ways. However, a more detailed inspection of the different ERP patterns reveals that there are important similarities. Patient 4 showed a large asymmetry, with the updating effect being significantly smaller for rightward compared with leftward trials over the medial frontocentral and centroparietal cortex. The pattern in Patient 2 is similar. Although the size of the updating effect was generally reduced, there was a significant asymmetry over the medial centroparietal cortex. As in Patient 4, the updating effect was smaller for rightward compared with leftward trials. Thus, in both patients, the updating effect is reduced when the first saccade is directed contralateral to the side of the lesion. This ERP pattern corresponds to the contralateral deficit in using corollary discharge information observed in the same patients in a previous behavioural study (Bellebaum et al., 2005a).

Patient 3 showed normal updating effects but a different topography than control subjects. Despite interindividual variability in control subjects, the right hemisphere is consistently more strongly involved than the left hemisphere when leftward saccades are performed, whereas both hemispheres are involved in updating for rightward saccades. The pattern in Patient 3 differs from the pattern in control subjects. He showed larger involvement of the right hemisphere, irrespective of the direction of the first saccade. Thus, in rightward trials, i.e. when the first saccade was directed away from the lesion side, the left hemisphere of Patient 3 was less involved in updating than in control subjects. Taken together, the ERP patterns of Patients 2, 3 and 4 can be interpreted in terms of insufficient relay of updating information because of the left thalamic lesion.

Consistent with the ipsilateral updating impairment following a left-sided MD lesion, the size of the updating effect in Patient 1 was reduced for leftward trials over the left frontocentral cortex. Furthermore, the topography of the updating effect was altered over the frontal cortex. As in Patient 3, the right hemisphere was more involved in updating than the left hemisphere, irrespective of the direction of the first saccade. However, in contrast to Patient 3, the left hemisphere in Patient 1 is ipsilateral to the observed behavioural deficit. It seems that in Patient 1, MD provides the ipsilateral hemisphere with information about ongoing saccades to the ipsilateral side and a lesion in MD thus leads to a reduction in the ipsilateral updating effect. When saccades are directed to the right, a sufficient amount of information about the saccade appears to be relayed.

\section{Size of deficits and alternative pathways}

In all thalamic lesion patients, the behavioural deficits found in the saccadic double-step task are relatively small (see Fig. 1). This is reflected in small processing differences compared with controls. Effects were observed only at a few electrode sites. Partially preserved updating-related processing in the patients appears to reflect reduced saccade-related information arriving in the cortex. Behavioural and ERP data thus seem to suggest that some saccade-related information is still relayed to the cortex via alternative pathways not affected by the thalamic lesion. Such pathways might involve the non-affected thalamic nuclei, e.g. the intralaminar nuclei of the central thalamus, which receive afferents from superior colliculus (Benevento \& Fallon, 1975 ) and project to the FEF and PPC (Kaufman \& Rosenquist, 1985). Corollary discharge information might also originate in the FEF, from where it is relayed to the PPC. It is also possible that corollary discharge information is relayed in the thalamus on the non-affected side, i.e. for the VL-lesioned patients the side ipsilateral to the saccade direction, possibly through MD. Although single cells in the monkey MD nearly exclusively discharge time-locked to contralateral saccades (Sommer \& Wurtz, 2004a) and temporal inactivation of the monkey MD leads to a deficit in processing corollary discharge for contralateral saccades, an ipsilateral deficit can be observed in individual cases (Sommer \& Wurtz, 2004b). Similarly, post-saccadic activity in FEF neurones has been shown to carry information about the intervening saccade in a double-step task, with nearly half of the neurones firing after ipsilateral saccades (Goldberg \& Bruce, 1990). As described above, the behavioural performance of Patient 1 may provide first evidence for a role of the human MD in relaying information about saccades to the ipsilateral side (see Bellebaum et al., 2005a). Although selective thalamic lesions are sparse, a replication of this observation in a larger sample is desirable, before firm conclusions can be reached. Similar to Patients 2, 3 and 4, alternative pathways in Patient 1 providing the cortex with saccade-related information may involve the non-affected right thalamus and intact nuclei of the left thalamus.

\section{Possible effects of the lesion-test interval}

As outlined above, ERP assessment occurred several months after saccade accuracy data had been obtained. Although saccade accuracy was not recorded in the present study, it is more than likely that the behavioural deficits in the patients persisted. The lesions in the four patients were acquired on average more than 6 years before the initial saccade accuracy assessment and were thus well after the acute phase of recovery. It is unlikely that additional recovery occurred in the few months between the first study and the present ERP assessment.

The ERP patterns of the patients in the present study may, however, at least partly reflect reorganization processes. For example, the updating-related ERP patterns of Patients 2, 3 and 4 do share some features but also differ to some degree, although the lesions affected nucleus VL of the left thalamus in all three patients and their behavioural deficits are very similar. In compensating for the loss of information about ongoing saccades, different alternative pathways may have been recruited for the relay of saccade-related information to the cortex in different patients, leading to distinct ERP patterns. Recent evidence on updating across hemifields in split-brain monkeys 
suggests that updating is mediated by a dynamic neural circuit (Berman et al., 2005; Heiser et al., 2005). In the intact monkey, updating across hemifields appears to be mediated by cortico-cortical connections. Transection of the corpus callosum and the anterior commissure leads to behavioural deficits but split-brain monkeys show rapid recovery (within days) of behaviour (Berman et al., 2005), which is reflected in the firing pattern of lateral intraparietal area (LIP) neurones and may be mediated by subcortical pathways (Heiser et al., 2005). Behavioural as well as neurophysiological data also indicate that the reorganization processes following the same lesion can be different in different monkeys (Berman et al., 2005; Heiser et al., 2005).

\section{Nature of the updating effect in a double-step task}

The ERP results from the patients in the present study have implications for the interpretation of the updating effect described in a recent ERP study. In accordance with results obtained in healthy controls of the present study, updating in the PPC was reported to occur after the first saccade in a double-step task in humans (Bellebaum et al., 2005b). When monkeys performed a similar task, the majority of LIP neurones discharged only if the next saccade was directed into their receptive or movement field. In the intersaccade interval, activity shifted from the neurones coding for the direction of the first saccade to neurones coding for the direction of the second saccade (Mazzoni et al., 1996). It was suggested that activity in LIP is related to motor intention (Snyder et al., 2000). LIP neurones have been described to code for the direction of the next intended saccade (Snyder et al., 1997), even if the saccade is not executed (Bracewell et al., 1996). The timing of the post-saccadic parietal ERP wave thus seems to suggest that it is related to motor intention for the second saccade but it may also be related to memory of stimulus location (see next section).

Apart from reflecting motor intention or stimulus memory, two observations led us to assume that the ERP wave primarily contains information about the first saccade. First, the component was more pronounced when ERPs were time-locked to first saccade onset compared with second saccade onset. Second, asymmetries in the parietal sources for leftward and rightward first saccades were observed. The ERP patterns observed in the thalamic lesion patients of the present study support this view. As revealed by behavioural evidence, information about the first saccade in the double-step task is unilaterally reduced in the patients and updating-related processing changes reflect this reduction.

Although the timing of the updating effect may also be compatible with other sources of information, such as proprioception, saccaderelated information seems to be provided by a corollary discharge signal of the saccadic movement command. Although proprioception plays a role in providing spatial constancy, corollary discharge is much more important (Bridgeman, 1995; Weir, 2000). Post-saccadic ERP components in earlier studies have also been attributed to incoming corollary discharge or efference copy information in the parietal cortex (Skrandies \& Laschke, 1997; Anagnostou et al., 2000; Kleiser \& Skrandies, 2000). Furthermore, anatomical, physiological and behavioural evidence indicates that the thalamus relays efference copy information about saccades (Sommer \& Wurtz, 2004a,b), so that the altered updating effect of patients in the present study is likely to reflect a reduced amount of corollary discharge information. The postsaccadic ERP wave associated with updating observed in healthy control subjects of the present study and in a previous ERP study (Bellebaum et al., 2005b) therefore seems to reflect the integration of corollary discharge information about the first saccade and the planning of the second saccade.

\section{Updating in other tasks}

Instead of being related to motor intention, the positive ERP wave observed in the present study might also reflect a response to the second target stimulus in the double-step task. In LIP and the FEF, single neurones have been shown to respond to the memory trace of an already vanished stimulus in the post-saccadic receptive field with latencies resembling the latency of the updating component in the present study (Duhamel et al., 1992a; Umeno \& Goldberg, 2001). However, many of these neurones also show predictive remapping (Umeno \& Goldberg, 2001; Kusunoki \& Goldberg, 2003). In both LIP and FEF, the average response latency for the 'memory response' is shorter than the typical visual on-response. Thus, the updating ERP component of the present study would be expected to occur earlier if it reflected a visual memory response. However, the findings of the present study do not necessarily contradict the finding of predictive remapping.

First, the onset of the parietal updating effect is about $150 \mathrm{~ms}$ after onset of the first saccade. A non-predictive response to the remapped location of the second target stimulus would be expected at about $180 \mathrm{~ms}$ after first saccade offset because, in the fixation task, the most pronounced VEP was observed at about $180 \mathrm{~ms}$ following target onset. Thus, it is possible that the updating effect, at least in part, reflects predictive remapping of the location of the second target stimulus.

Second, the timing of cortical processing related to updating may depend on the specific task requirements. In most studies showing predictive remapping only one saccade was required and the to-beupdated stimulus was presented shortly before the saccade. In ERP studies, in which stimuli were presented during or before a single saccade, positive signals starting between 60 and $120 \mathrm{~ms}$ after saccade onset were discussed in terms of an incoming corollary discharge signal in occipito-parietal cortex (Skrandies \& Laschke, 1997; Anagnostou et al., 2000; Kleiser \& Skrandies, 2000; Bellebaum \& Daum, 2006). This component was even observed without visual stimulation (Skrandies \& Laschke, 1997).

In a double-step task, information about the first saccade is needed when the second saccade is planned. Transcranial magnetic stimulation pulses applied over the PPC disrupt performance when they are applied $150 \mathrm{~ms}$ after first saccade onset but not when applied earlier (van Donkelaar \& Müri, 2002). In accordance with this finding, the data of the present study suggest that corollary discharge information is put into functional use before the second saccade in a double-step task. In other tasks, corollary discharge processing may occur earlier.

\section{Conclusions}

The present study shows that thalamic lesions are associated with altered updating-related cortical processing in humans. Processing changes in thalamic lesion patients are consistent with the hypothesis that the thalamus relays corollary discharge information about saccadic eye movements to the cortex. This finding also sheds new light on the parietal updating process in saccade sequences. Corollary discharge information about a just-executed saccade is integrated with motor-intention-related programming and/or with retinal information about stimulus location in the PPC directly before the next saccade occurs. 


\section{Acknowledgements}

This work was funded by the International Graduate School of Neuroscience of the Ruhr-University of Bochum, Germany.

\section{Abbreviations}

CC, control condition; ERP, event-related potential; FEF, frontal eye field; LIP, lateral intraparietal area; MD, medio-dorsal nucleus of the thalamus; PPC, posterior parietal cortex; RDC, retino-spatial dissonance condition; VEP, visual evoked potential; VL, ventrolateral nucleus of the thalamus.

\section{References}

Anagnostou, E., Kleiser, R. \& Skrandies, W. (2000) Electrophysiological correlates of human intrasaccadic processing. Exp. Brain Res., 130, 177187.

Bellebaum, C. \& Daum, I. (2006) Time course of cross-hemispheric spatial updating in the human parietal cortex. Behav. Brain Res., 169, $150-161$.

Bellebaum, C., Daum, I., Koch, B., Schwarz, M. \& Hoffmann, K.P. (2005a) The role of the human thalamus in processing corollary discharge. Brain, $128,1139-1154$.

Bellebaum, C., Hoffmann, K.P. \& Daum, I. (2005b) Post-saccadic updating of visual space in the posterior parietal cortex in humans. Behav. Brain Res., 163, 194-203.

Benevento, L.A. \& Fallon, J.H. (1975) The ascending projections of the superior colliculus in the rhesus monkey (Macaca mulatta). J. Comp. Neurol., 160, 339-361.

Berman, R.A., Heiser, L.M., Saunders, R.C. \& Colby, C.L. (2005) Dynamic circuitry for updating spatial representations. I. Behavioral evidence for interhemispheric transfer in the split-brain macaque. J. Neurophysiol., 94, 3228-3248.

Bracewell, R.M., Mazzoni, P., Barash, S. \& Andersen, R.A. (1996) Motor intention activity in the macaque's lateral intraparietal area. II. Changes of motor plan. J. Neurophysiol., 76, 1457-1464.

Bridgeman, B. (1995) A review of the role of efference copy in sensory and oculomotor control systems. Ann. Biomed. Eng., 23, 409-422.

Colby, C.L. \& Goldberg, M.E. (1999) Space and attention in parietal cortex. Annu. Rev. Neurosci., 22, 319-349.

van Donkelaar, P. \& Müri, R. (2002) Craniotopic updating of visual space across saccades in the human posterior parietal cortex. Proc. R. Soc. Lond. B Biol. Sci., 269, 735-739.

Duhamel, J.R., Colby, C.L. \& Goldberg, M.E. (1992a) The updating of the representation of visual space in parietal cortex by intended eye movements. Science, 255, 90-92.

Duhamel, J.R., Goldberg, M.E., Fitzgibbon, E.J., Sirigu, A. \& Grafman, J. (1992b) Saccadic dysmetria in a patient with a right frontoparietal lesion. The importance of corollary discharge for accurate spatial behaviour. Brain, 115 (5), 1387-1402.

Gaymard, B., Rivaud, S. \& Pierrot-Deseilligny, C. (1994) Impairment of extraretinal eye position signals after central thalamic lesions in humans. Exp. Brain Res., 102, 1-9.

Goldberg, M.E. \& Bruce, C.J. (1990) Primate frontal eye fields. III. Maintenance of a spatially accurate saccade signal. J. Neurophysiol., 64, 489-508.

Gratton, G., Coles, M.G. \& Donchin, E. (1983) A new method for off-line removal of ocular artifact. Electroencephalogr. Clin. Neurophysiol., 55, 468484.

Haarmeier, T., Thier, P., Repnow, M. \& Petersen, D. (1997) False perception of motion in a patient who cannot compensate for eye movements. Nature, $\mathbf{3 8 9}$, 849-852.

Hallett, P.E. \& Lightstone, A.D. (1976) Saccadic eye movements to flashed targets. Vision Res., 16, 107-114.

Heide, W. \& Kompf, D. (1998) Combined deficits of saccades and visuo-spatial orientation after cortical lesions. Exp. Brain Res., 123, 164-171.

Heide, W., Binkofski, F., Seitz, R.J., Posse, S., Nitschke, M.F., Freund, H.J. \& Kompf, D. (2001) Activation of frontoparietal cortices during memorized triple-step sequences of saccadic eye movements: an fMRI study. Eur. J. Neurosci., 13, 1177-1189.
Heiser, L.M., Berman, R.A., Saunders, R.C. \& Colby, C.L. (2005) Dynamic circuitry for updating spatial representations. II. Physiological evidence for interhemispheric transfer in area LIP of the split-brain macaque. J. Neurophysiol., 94, 3249-3258.

von Holst, E. \& Mittelstaedt, H. (1950) Das Reafferenzprinzip (Wechselwirkungen zwischen Zentralnervensystem umd Peripherie). Naturwissenschaften, 37, 464-476.

Huerta, M.F., Krubitzer, L.A. \& Kaas, J.H. (1987) Frontal eye field as defined by intracortical microstimulation in squirrel monkeys, owl monkeys, and macaque monkeys. II. Cortical connections. J. Comp. Neurol., 265, 332-361.

Kaufman, E.F. \& Rosenquist, A.C. (1985) Efferent projections of the thalamic intralaminar nuclei in the cat. Brain Res., 335, 257-279.

Kleiser, R. \& Skrandies, W. (2000) Neural correlates of reafference: evoked brain activity during motion perception and saccadic eye movements. Exp. Brain Res., 133, 312-320.

Kusunoki, M. \& Goldberg, M.E. (2003) The time course of perisaccadic receptive field shifts in the lateral intraparietal area of the monkey. J. Neurophysiol., 89, 1519-1527.

Mai, J.K., Assheuer, J. \& Paxinos, G. (1997) Atlas of the Human Brain. Academic Press, San Diego.

Mazzoni, P., Bracewell, R.M., Barash, S. \& Andersen, R.A. (1996) Motor intention activity in the macaque's lateral intraparietal area. I. Dissociation of motor plan from sensory memory. J. Neurophysiol., 76, 1439-1456.

Medendorp, W.P., Goltz, H.C., Vilis, T. \& Crawford, J.D. (2003) Gazecentered updating of visual space in human parietal cortex. J. Neurosci., 23, 6209-6214.

Rugg, M.D., Doyle, M.C. \& Wells, T. (1995) Word and nonword repetition within- and across-modality: an event-related potential study. J. Cogn. Neurosci., 7, 209-227.

Sapir, A., Hayes, A., Henik, A., Danziger, S. \& Rafal, R. (2004) Parietal lobe lesions disrupt saccadic remapping of inhibitory location tagging. J. Cogn. Neurosci., 16, 503-509.

Sereno, M.I., Pitzalis, S. \& Martinez, A. (2001) Mapping of contralateral space in retinotopic coordinates by a parietal cortical area in humans. Science, 294, $1350-1354$

Skrandies, W. \& Laschke, K. (1997) Topography of visually evoked brain activity during eye movements: lambda waves, saccadic suppression, and discrimination performance. Int. J. Psychophysiol., 27, 15-27.

Snyder, L.H., Batista, A.P. \& Andersen, R.A. (1997) Coding of intention in the posterior parietal cortex. Nature, 386, 167-170.

Snyder, L.H., Batista, A.P. \& Andersen, R.A. (2000) Intention-related activity in the posterior parietal cortex: a review. Vision Res., 40, 1433-1441.

Sommer, M.A. \& Wurtz, R.H. (2002) A pathway in primate brain for internal monitoring of movements. Science, 296, 1480-1482.

Sommer, M.A. \& Wurtz, R.H. (2004a) What the brain stem tells the frontal cortex. I. Oculomotor signals sent from superior colliculus to frontal eye field via mediodorsal thalamus. J. Neurophysiol., 91, 13811402 .

Sommer, M.A. \& Wurtz, R.H. (2004b) What the brain stem tells the frontal cortex. II. Role of the SC-MD-FEF pathway in corollary discharge. J. Neurophysiol., 91, 1403-1423.

Sperry, R.W. (1950) Neural basis of the spontaneous optokinetic response produced by visual inversion. J. Comp. Physiol. Psychol., 43, 482-489.

Stanton, G.B., Bruce, C.J. \& Goldberg, M.E. (1995) Topography of projections to posterior cortical areas from the macaque frontal eye fields. J. Comp. Neurol., 353, 291-305.

Thickbroom, G.W. \& Mastaglia, F.L. (1985) Cerebral events preceding selfpaced and visually triggered saccades. A study of presaccadic potentials. Electroencephalogr. Clin. Neurophysiol., 62, 277-289.

Tobler, P.N., Felblinger, J., Burki, M., Nirkko, A.C., Ozdoba, C. \& Müri, R.M. (2001) Functional organisation of the saccadic reference system processing extraretinal signals in humans. Vision Res., 41, 13511358.

Umeno, M.M. \& Goldberg, M.E. (2001) Spatial processing in the monkey frontal eye field. II. Memory responses. J. Neurophysiol., 86, 2344-2352.

Versino, M., Beltrami, G., Uggetti, C. \& Cosi, V. (2000) Auditory saccade impairment after central thalamus lesions. J. Neurol. Neurosurg. Psychiat., 68, 234-237.

Weir, C.R. (2000) Spatial localisation: does extraocular muscle proprioception play a role? Graefes Arch. Clin. Exp. Ophthalmol., 238, 868-873. 